\title{
Receptive-field Size of S1 Cortical Neurones is Altered by Methaqualone via a GABA Mechanism
}

\author{
T.P. Hicks, T. Kaneko and J.-I. Oka
}

\begin{abstract}
Methaqualone (Mtq; quaaludes or 'ludes) is a controlled substance, having a molecular structure related to the imidiazobenzodiazepine series of drugs, that has gained some notoriety recently due to its history of widespread abuse on the street. Users report experiencing peripheral paresthesia and transient numbness on body parts receiving dense cutaneous innervation (lips, fingertips, etc.). Since the receptive-field (RF)-sizes of many primary somatosensory (S1) cortical neurones are controlled by local, gamma-aminobutyric acid (GABA)-mediated inhibitory processes, we tested Mtq to see whether its clinical symptoms might have a basis in an action through central GABA-mediated synaptic processes. This report supports this contention and describes a likely pharmacological mechanism involved as one being related to the Ro 15-1788-sensitive benzodiazepine (Bzd) recognition site(s) of the GABA receptor complex.

RÉSUMÉ: La taille du champ réceptif des neurones corticaux S1 est modifiée par la méthaqualone via un mécanisme gabaergique La méthaqualone (Mtq; quaaludes ou 'ludes) est une substance contrôlée qui a une structure moléculaire reliée aux drogues de la famille des imidiazobenzodiazépines et qui a acquis une certaine notoriété récemment à cause d'abus largement répandus chez les usagers de drogues. Ils rapportent éprouver des paresthésies périphériques et des engourdissements transitoires des parties du corps pourvues d'une innervation cutanée dense (les lèvres, le bout des doigts etc.). Comme la taille du champ réceptif de plusieurs neurones corticaux somesthésiques primaires ( $\mathrm{S} 1$ ) est contrôlée par des processus inhibiteurs dont la médiation se fait par l'acide gamma-aminobutyrique (GABA), nous avons vérifié si les symptômes cliniques de la Mtq pourraient être en relation avec un mode d'action faisant appel aux processus synaptiques centraux à médiation GABAergique. Ce compte-rendu supporte cette affirmation et décrit un mécanisme pharmacologique vraisemblablement impliqué comme étant relié au( $\mathrm{x}$ ) sites de reconnaissance des benzodiazépines du complexe récepteur GABA qui est sensible au Ro 15-1788.
\end{abstract}

Can. J. Neurol. Sci. 1989; 17: 30-34

Methaqualone (Mtq) is a 2,3-disubstituted quinazolinone having anticonvulsant actions, that has been employed clinically to treat insomnia because it possesses sedative and hypnotic properties. ' Dose-related changes in the drug's direction of effect have been reported on stage 4 and REM sleep;2.3 at high doses it is known to depress spinal polysynaptic pathways. ${ }^{4}$ When administered at doses effective in producing a somnolescent or hypnotic state, abnormal cutaneous sensibility (numbness and tingling as well as some loss of motor ability) is experienced; 5 these symptoms may persist beyond the initial treatment. ${ }^{6}$

Air-puffer stimulation of cutaneous receptive fields (RFs) in non-human mammalian species provides a reliable and reproducible method of driving many low-threshold, mechanoreceptive primary somatosensory (S1) cortical cells. ${ }^{7}$ Microiontophoretic ejection of low doses of gamma-aminobutyric acid (GABA) elevates thresholds of $S 1$ neurones to activation by cutaneous stimuli and either decreases RF size or eliminates activation by peripheral stimuli altogether ${ }^{8-10}$ These effects are reversed by concurrent ejection of the $\mathrm{GABA}_{\mathrm{A}}$ selective receptor antagonist, bicuculline methiodide (BMI). When delivered alone to cortical neurones, BMI reduces response thresholds to tactile stimulation of RFs and orthodromic synaptic activation following VPL stimulation." Simultaneously it produces dramatic enlargements of neuronal RF size to dimensions severalfold greater than observed prior to the drug's administration. $8-11$ The changes observed with GABA apply to cells in all functional subtypes of cortex, whereas the RF-enlarging action of BMI is restricted to cells in rapidly adapting, but not in slowly adapting or low-velocity, rapidly adapting areas.

It might be expected that threshold elevations and RF size diminutions of SI cortical cells would lead to altered sensory

From the Department of Psychology, University of North Carolina at Greensboro, Greensboro, North Carolina

Received March 15, 1989. Accepted in final form October 6, 1989

Reprint requests to: Dr. T.P. Hicks, Department of Psychology, University of North Carolina at Greensboro, 296 Eberhart Building, Greensboro, N.C., U.S.A. $27412-5001$ 
experience - such as numbness and paresthesia - as those changes in single cell response properties may be predicted to contribute to a decrease of somatic perceptual awareness. Since the RF size of rapidly adapting $S 1$ neurones is controlled by GABA-mediated, intracortical inhibition, $8-10$ tests with Mtq were initiated in anaesthetized cats using local, iontophoretic administration and systemic administration (i.p. injections) of Mtq, to explore the possibility that the paresthesias reported by users of the drug are related directly to central actions on neuronal RF size and threshold. Our results support this proposition and show further that: 1) the effects of Mtq are synergistic with those of GABA on the variables of RF size, cell threshold to natural activation of the cutaneous surface and responsiveness to synaptic driving by electrical stimulation of the thalamus, and 2) the enhancements of Mtq of intrinsic, GABA-mediated inhibitions are reversed by the Bzd receptor antagonist, Ro 15-1788.12

\section{Materials and MeThods}

Cats were anaesthetized with intraperitoneal (i.p.) injections of pentobarbital sodium ( $35 \mathrm{mg} / \mathrm{kg}$ ) and maintained with supplementary i.v. doses of the same anaesthetic to mean values ranging between 1.8 and $3.0 \mathrm{mg} / \mathrm{kg} \mathrm{h}^{-1}$. After preparation for electrophysiological recording using standard procedures, data were collected from single neurones in the forelimb representation of SI cortex with carbon fibre-filled, multibarrel micropipette electrodes. Cells were classified according to their threshold of activation from the cutaneous surface, their response to stimulation of the nucleus ventroposterior lateralis (VPL) and to the nature of the background discharge elicited by tactile stimulation of the skin in and around the area of the RF.8-11

Electrode penetrations were oriented normal to the pial surface and were made through the cortical region where cells rep- resenting the forepaw and forelimb are located. A 6-jet air puffer $7,13,14$ was used to stimulate the RFs of cells. This device provides a reliable and reproducible, quantitatively-controlled method of driving low threshold mechanoreceptive cortical cells having cutaneous and/or hair RFs. The cutaneous surface first was examined carefully with hand-held probes to search for excitatory RFs and to determine their size. Air puffer jets then were placed 1 to $5 \mathrm{~mm}$ from the skin's surface at loci corresponding to central and peripheral subregions of the RF. Tests were made through each channel separately (Figure 1). In the combined trials (puffers 1+2; dotted bar in Figure 1), half the control air pressure was delivered through each channel activated concurrently, so as to deliver the same total puff of air, but distributed over a wider area to test for possible spatial summation-specific effects of drugs. This procedure was justified empirically by observing and comparing responses of $S 1$ cells in two conditions: 1) by a standard air puff through one jet alone and 2) by combined stimulation using two jets, activated concurrently, each at half pressure, having their tips pointed to neighboring sites within the cell's cutaneous RF. The responses elicited by these two different means always were identical in magnitude during such tests.

The following substances in solution were contained within the drug barrels of the micropipette assemblies and all were ejected as cations: GABA (0.5 M, pH 3.5), BMI $(5 \mathrm{mM}$ in $0.9 \% \mathrm{NaCl}, \mathrm{pH} 3.3$ ), $\mathrm{Mtq} \mathrm{HCl}$ and Ro $15-1788$ (both $2.5 \mathrm{mM}$ in $0.9 \% \mathrm{NaCl}, \mathrm{pH} 3.2$ ); the central barrel of the assembly was used for extracellular recording through the carbon fibre.

\section{RESULTS}

Mtq (as well as GABA) at low doses (20 to $80 \mathrm{nA} ; \overline{\mathrm{x}}=35$ $n A, n=13$ cells) consistently suppressed responses of cortical

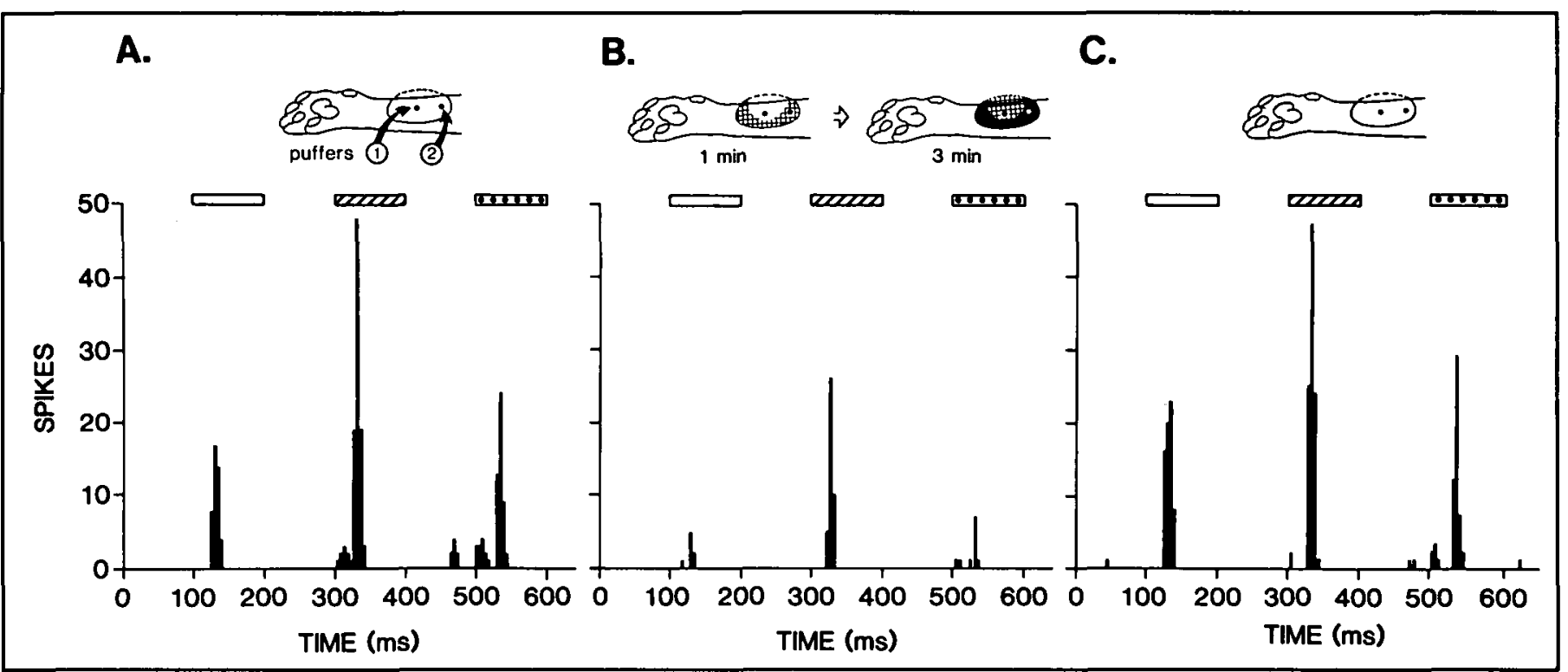

Figure I - Peristimulus time histograms obtained from responses of a cell activated at short latency (2.3 ms) following VPL stimulation, and located at a depth of $1037 \mu \mathrm{m}$ in a region of cortex characterized by rapidly adapting multiunit activity. The RF was stimulated by the air-puffer at iwo locations as shown in the inserted drawings. Puffers $I$ and 2 correspond to the diagonal bars and open bars along the PSTH abscissae, resp. The outer boundary described on the drawing of the forelimb indicates the control RF. Hatched area indicates region of elevated threshold to punctate stimulation. Blackened area of $R F$ indicates region of total tactile insensitivity. Response to first puffer seen beneath open bar; to puffer 2, beneath diagonally-striped bar; to combined (puffers I + 2) stimulation, beneath bar containing filled circles. A: Control (no drugs). B: During Mtq ejection, 50 nA. C: Recovery from effects of Mtq. 
cells to peripheral stimulation, depressed spontaneous firing, elevated threshold to synaptic (VPL) driving and often produced a reduction of RF size, irrespective of the rate of adaptation of the multiunit activity in which the cell was situated, or upon method of drug administration (i.p. injection at 20 to $40 \mathrm{mg} / \mathrm{kg}$ or iontophoretic ejection) (Figure 1). Accuracy of determination of RF boundaries was ensured by assessments made independently by each of three investigators upon the same RFs and comparing each different evaluation. This procedure always produced RF size measurements within margins of error that rarely exceeded 1-2 mm for any given point on the RF perimeter. For the responses of the cell illustrated by the example of Figure 1, during iontophoretic expulsion of Mtq (50 nA, 1 to $3 \mathrm{~min}$ ) the threshold to cutaneous stimulation was elevated (first inset drawing of Figure 1B: hatched area only). This change was measured in both central and peripheral subregions of the RF. The amplitude of the puffer-elicited response in the peripheral subregion (puffer 2: open bar in PSTH) was reduced to just $17 \%$ of the control value, while that elicited from the more centrally located puffer (puffer 1: diagonally hatched bar in PSTH) was reduced to $43 \%$ of control. During this sequence, stimulation of the cell's RF with hand-held probes showed that the threshold in the central subregion of the RF had changes from low to medium (defined as being a change to a mean value of $>25 \%$ reduction in evoked response) whereas in the peripheral subregion of the RF, it went from low to high (defined as being a change to a mean value of $>75 \%$ reduction in evoked response; hatching, left in B). Responses resulting from the spatially distributed stimulus (dotted bar in PSTH), produced by a combined activation of puffers 1 and 2, each puff being delivered at half the normal pressure, showed a reduction to $21 \%$ of control; spontaneous activity of the cell was also abolished (all percentage values refer to total number of cell responses (spikes) for 30 stimulation sequences and these values are usually the means of 3 or 4 separate determinations). Response variability among determinations was $<5 \%$, where one determination equals the total number of spikes elicited following 30 stimulation sequences. With maintained administration of Mtq (after $3 \mathrm{~min}$ at $50 \mathrm{nA}$ ) the RF size decreased so that stimulation of the region of elevated threshold shown in Figure 1B (blackened area of inset figure) no longer produced a response (not illustrated). Following cessation of ejection of Mtq (Figure 1C), pufferelicited responses returned within three min to values similar to the control: puffer $1,109 \% ; 2,100 \%$ and $1+2,96 \%$. The RF size also recovered.

For the remaining cells tested in this experimental series, the threshold for a response to cutaneous stimulation was elevated most in the periphery of the cells' RF. Responses elicited by a stimulus covering most of the cells' RF, invoking both peripheral (RF surround) and central (RF centre: "hot spot") excitatory mechanisms were reduced to intermediate levels, compared to the reductions observed following stimulation of the central or peripheral sites alone. Ejections of Mtq with higher currents (eg, 80 to $200 \mathrm{nA}$ ) or prolonged drug administration (>15 to $20 \mathrm{~min}$ at 20 to $80 \mathrm{nA}$ ) usually produced further diminutions of RF size. All effects were reversed upon cessation of ejection of drugs. Similar results to those illustrated by Figure 1, using doses between 20 and $200 \mathrm{nA}$ ( 5 to $20 \mathrm{~min}$ ejections), have been observed at an additional 12 neurones irre- spective of the nature of the prevailing multiunit evoked background activity, at recording depths ranging from 578 to $1795 \mu \mathrm{m}$ from the pial surface. I.p. injection of Mtq in two other cases (at $40 \mathrm{mg} / \mathrm{kg}$ ) produced identical results to those observed with iontophoretic delivery, during a period of observation encompassing 15 to 45 min post-injection. Recovery was not observed in these latter cases. The effects of iontophoretic Mtq were of slow onset, requiring on the order of minutes to occur, rather than of seconds as was the case with GABA. However, the relatively poor solubility of this substance ${ }^{4}$ combined with its reduced mobility in a solution of low ionic strength could contribute to this slower time course. ${ }^{15}$

The effects of Mtq were examined in the presence of substances (see Methods) active at GABA or Bzd receptors, and interactions using different combinations of drug administration, as described below, were noted on the response properties of the cells tested. One striking result was the finding that GABA and Mtq exerted synergistic actions, as opposed to additive ones, upon RF size. This finding was obtained at each of the 12 neurones tested.

The example illustrated in Figure $2 \mathrm{~A}$ shows that when GABA and Mtq were delivered together, each at doses which previously had been determined to evoke submaximal effects, the resultant RF size was diminished to a degree much greater than the area that would be expected, based on an arithmetic sum of the effects upon RF area of each drug alone. The spatial extent of the control RF was determined prior to all drug tests and is illustrated in the top panel of Figure 2A (RF 1). Ejection of $\mathrm{Mtq}$ at $80 \mathrm{nA}$ for 5 to $10 \mathrm{~min}$, in addition to producing an elevation of threshold, effected a $15 \%$ reduction of RF size (RF 2). When GABA was tested alone, an ejection of $3 \mathrm{nA}$ produced diminutions of RF size that were relatively greater than those seen with Mtq, for drug administration times of $5 \mathrm{~min}$ (RF 3) and $10 \mathrm{~min}$ (RF 4). While maintaining ejection of GABA at $3 \mathrm{nA}$, Mtq again was delivered to $80 \mathrm{nA}$ but this time, simultaneous administration of $3 \mathrm{nA}$ of GABA resulted in a final RF size after $5 \mathrm{~min}$ (RF 5) and most notably after $10 \mathrm{~min}$ (RF 6), which was diminished much more than was expected on a linear basis from that seen when either of the drugs was delivered alone (expected arithmetic interaction was $42 \%$ of control RF; actual size measured was $<2 \%$ ). Fifteen min after stopping the Mtq ejection, the GABA-suppressed RF dimension recovered to pre-Mtq size (lower panel in Figure 2A: RF 7). The RF returned essentially to control size when the GABA ejecting current was terminated (RF 8).

In Figure 2B, the control RF again is indicated as RF 1. Initially, ejection of $100 \mathrm{nA}$ of Mtq brought about a $40 \%$ reduction of RF size after 10 min (RF 2). The effect of Ro 15-1788 alone ( $20 \mathrm{nA})$ then was assessed and found to enlarge RF size to $120 \%$ of normal (RF 3: central panel of Figure 2B); during this time it was also observed that the the threshold to natural stimulation was decreased markedly. Maintaining this ejection at its effective dose of $20 \mathrm{nA}$, an additional ejection of Mtq at $100 \mathrm{nA}$ was commenced and reductions of RF size occurred as shown for $5 \mathrm{~min}$ (RF 4) and $10 \mathrm{~min}$ (RF 5). Note that the ultimate RF shrinkage under the influence of Ro 15-1788 in combination with Mtq never reached the level achieved when Mtq was tested alone (RF 2) until the administration of Ro 15-1788 ceased (lower panel, Figure 2B: RF 6), supporting the view that these 
drug interactions are of a competitive nature and therefore that their likely mediation is via receptor mechanisms. At this point, Mtq again yielded a RF of the same size as observed prior to ejection of Ro 15-1788 (compare RF 6 with RF 2, upper and lower panels of Figure $2 \mathrm{~B}$ ). The original size of the cell's $\mathrm{RF}$ reappeared upon cessation of ejection of all drugs (RF 7). Similar results have been observed on each of 12 neurones so tested and the quantitative data from these iontophoretic experiments are presented in the normalized histograms of Figure 3 for 8 of these 12 cells tested.

A noteworthy but unexpected finding was the different effects of Mtq at different doses. Opposite actions of different doses have been observed with compounds which are members of the imidiazobenzodiazepine series (eg, midazolam), ${ }^{13}$ but not previously with quinazolinone derivatives. In 4 cases of iontophoretic delivery and in 2 additional cases using i.p. injections (10 and $20 \mathrm{mg} / \mathrm{kg}$ ), RF enlargements of up to 3 -fold normal were obtained with Mtq. These alterations all were accompanied

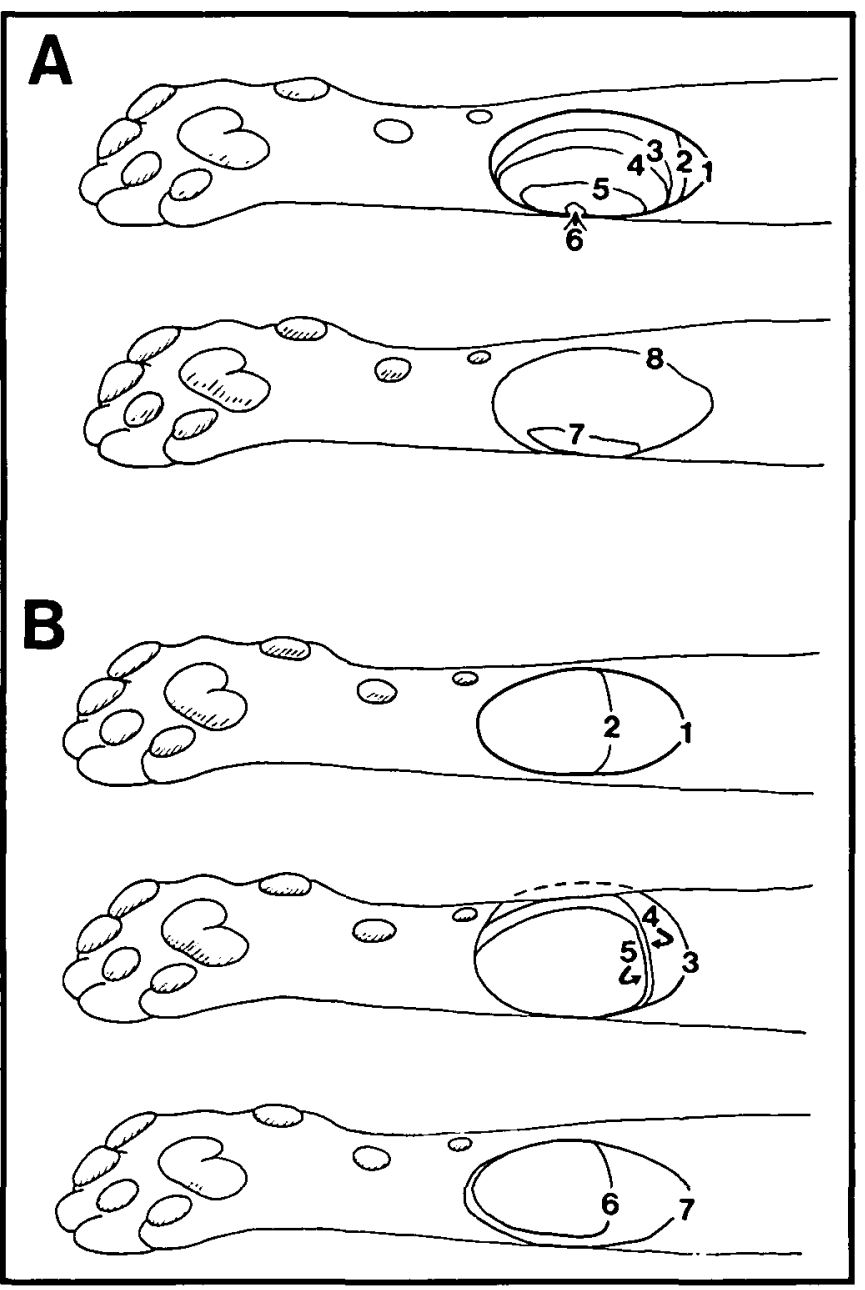

Figure $2-(A)$ Miq exerts synergistic effects upon RF size when tested with GABA. Responses obtained from a cell, in an area characterized by rapidly adapting multiunit activity, at a cortical depth of $940 \mu \mathrm{m}$, which was activated orthodromically (l.6 mis latency) yielded RF maps as indicated. (B) Effect of Ro 15-1788 on Miqinduced dimimutions of $R F$ size. Same cell provided the $R F$ maps as illustrated in Figure $2 A$. by a lowering of response threshold to VPL-evoked firing and to naturally elicited excitations and, in some cases, by increases in the rate of spontaneous firing (from a mean value of $0.8 \pm 0.2 \mathrm{~Hz}$, to $3.8 \pm 0.5 \mathrm{~Hz} ; \mathrm{n}=4$ ). In 3 of the iontophoretic tests when they were continued with delivery at higher doses (involving a doubling, or more, of the original dose to final doses ranging between 50 and $200 \mathrm{nA}$ ), these effects ceased and then reversed.

\section{Discussion}

The similarity between the effects of i.p. injections and iontophoretic administration of Mtq is important because it eliminates the possibility, 1) that in the latter case, the effects occur only with much higher concentrations of Mtq that might be delivered from the micropipette but that might not occur in the brains of drug users, and 2) that systemic Mtq, after absorption, is transformed to some other substance that is inactive at the level of the somatosensory cortex.

It has been shown that Bzd can modulate the RF-shaping actions of intrinsic, GABA-mediated inhibitions in $\mathrm{S} I$ cortex. ${ }^{16}$ In part due to the fact that Mtq bears a close structural similarity to many compounds of the Bzd series, it has been proposed 17 that Mtq acts, to some extent at least, specifically with the Bzd receptor site (as defined through displacement of $\left[{ }^{3} \mathrm{H}\right]$-diazepam binding). However, neurochemical and behavioural experiments have suggested that Mtq does not exert its effects solely through the Bzd receptor or via the chloride ionophore, but rather that it affects elements of the GABA receptor complex more globally. ${ }^{18,19}$ This issue concerning its mechanism of action has not been easy to resolve. Whereas the effects seen here appear to be

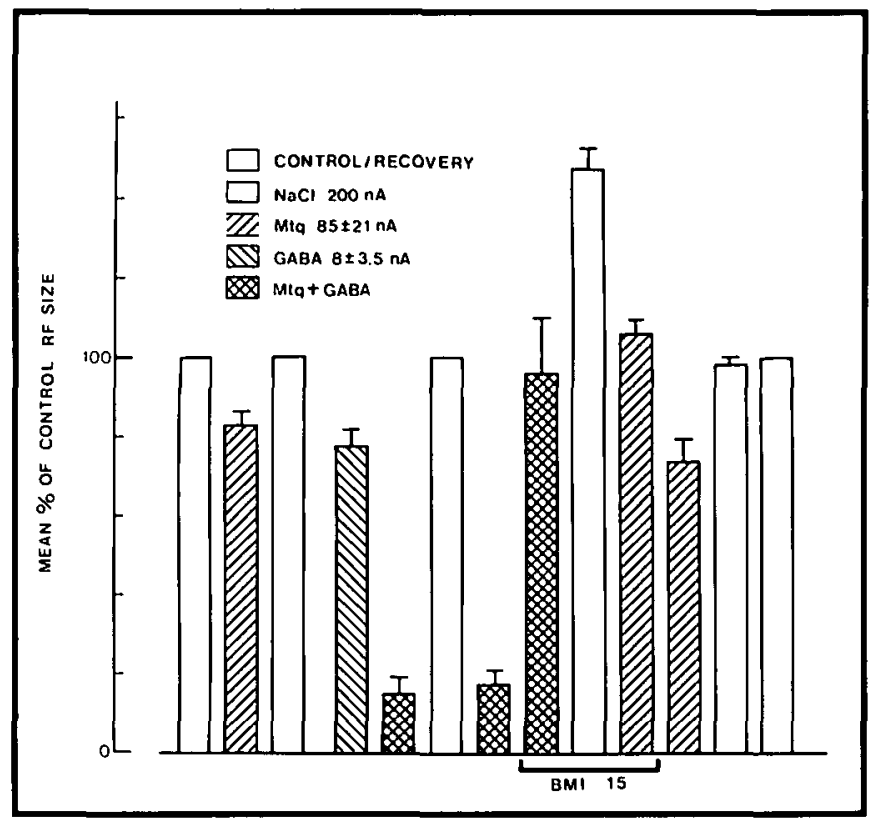

Figure 3 - Mean change in percent ( \pm S.E.M.) of control RF size in response to Mtq. GABA. Miq plus GABA alone and in the presence of $B M I$, and during ejection of $\mathrm{NaCl}$ as a current control, for 8 neurones where complete tests with all drugs were made using iontophoretic ejection. Control (pre-drug) RF size is expressed as $100 \%$ for each case, to permit the data to be compared in normalized fashion. 
receptor-linked due to the nature of the pharmacological interactions observed among the agonists and antagonists, it remains difficult, using the in vivo experimental design, to actually propose the specific underlying molecular mechanism responsible. The present protocol provides functional relevance to the effects of $\mathrm{Mtq}$, rather than simply a mechanistic one. The methodology employed here permits Mtq effects on cellular processes examined at the single unit level to be related to clinical observations; such relationships could not be achieved in more reduced preparations such as tissue slices or with neurones in culture. Using natural stimulation and microiontophoretic manipulation of RF size and measurements of response threshold of $\mathrm{S} 1$ cortical neurones, related compounds ${ }^{20}$ suspected of having pharmacological action at the GABA receptor complex also can be evaluated. Hence, the present approach may prove valuable in future studies of GABA receptor pharmacology, and to our understanding of certain types of synaptic integrative processes.

Considering the selectivity of action upon $\mathrm{S} 1$ response properties known to pertain to GABA, ${ }^{8-10}$ its agonists, ${ }^{7}$ antagonists ${ }^{8-}$ 10 and receptor modulators, $11,16,17$ we propose that the reported paresthesias attributed to Mtq are explained by its ability to elevate thresholds and reduce RF sizes. These effects appear to be mediated centrally by the post-synaptic influence of Mtq at, or very closely associated with, some component of the benzodiazepine recognition site of the GABA receptor complex. Thus, Mtq exerts Bzd-like synergism 16 with GABA on $\mathrm{S} 1$ neurones and likely therefore acts at this substituent of the GABA-Bzd receptor.

The RF size-decreasing action of Mtq was attenuated reversibly during ejection of the Bzd antagonist, Ro 15-1788 (Figure 2B); this finding emphasizes the similarity of effect of Mtq to that obtained with Bzd agonists ${ }^{13,16}$ and implicates the site of action as one that is linked to the Bzd recognition site(s). ${ }^{4 \cdot 12,17-20}$ Based on these data, it is tempting to offer the conjecture that this site may even be involved in some way with the sedative and hypnotic actions of Mtq, as might be expected of a drug that reduces somatic sensibility by RF-size diminution and threshold elevation. The midazolam-like action ${ }^{16}$ of $\mathrm{Mtq}$ recalls the dose-related effect described for the drug in its clinical activity profile upon stage 4 and REM sleep 2,3 in man.

The similarity of certain aspects of the effects of Mtq we observed at single neurones (reduction of tactile sensibility and depression of neuronal firing, for example) to that reported for certain features regarding the paresthesias experienced by human users of the drug 5,6 suggests that clinical investigators may in future utilize this potentially advantageous tool for predictive value in helping to judge the mode of action of allied substances.

\section{ACKNOWLEDGEMENTS}

We wish to acknowledge with gratitude the excellent technical assistance of Mrs. C.A. Stark, and the gift of methaqualone $\cdot \mathrm{HCL}$ from Dr. Michel Bhereur, Rougier Desbergers Inc. (Montreal). Drs. K. Kmjevic, E.G. Jones, R.W. Dykes and I. Parhad are thanked for critically reading earlier versions of the paper. This work was supported financially by the MRC of Canada and the Alberta Heritage Foundation For Medical Research.

\section{REFERENCES}

1. Brown SS, Goenechea S. Methaqualone: Metabolic, kinetic, and clinical pharmacologic observations. Clin Pharmacol Ther 1973; 14: 314-324.

2. Soulairac A, Gottesman C. Experimental studies on sleep produced by methaqualone. Life Sci 1967; 6: 1229-1232.

3. Williams RL, Agnew HW Jr. The effects of drugs on the EEG sleep patterns of normal humans. Exp Med Surg 1969; 27: 53-64.

4. Swift JG, Dickens EA, Becker BA. Anticonvulsant and other pharmacological activities of tuazolone. Arch Int Pharmacodyn Ther 1960; 128: 112-125.

5. Marks P. Methaqualone and peripheral neuropathy. Practitioner 1974; $212: 721-722$.

6. Hoaken PCS. Adverse effect of methaqualone. Can Med Assoc J 1975; $112: 685$.

7. Kaneko T, Hicks TP. Baclofen and $\gamma$-aminobutyric acid differentially suppress the cutaneous responsiveness of primary somatosensory cortical neurones. Brain Res 1988; 443: 360-366.

8. Hicks TP, Dykes RW. Receptive field size for certain neurons in primary somatosensory cortex is determined by GABA-mediated intracortical inhibition. Brain Res 1983; 274: 160-164.

9. Dykes RW, Landry P, Metherate R, et al. Functional role of GABA in cat primary somatosensory cortex: shaping receptive fields of cortical neurons. J Neurophysiol 1984; 52: 1066-1093.

10. Alloway $\mathrm{KD}$, Burton $\mathrm{H}$. Bicuculline-induced alterations in neuronal responses to controlled tactile stimuli in the second somatosensory cortex of the cat: a microiontophoretic study. Somatosens Res 1986; 3: 197-211.

11. Hicks TP, Landry P, Metherate R, et al. Functional properties of neurons mediated by GABA in cat somatosensory cortex under barbiturate and urethane anesthesia. In: Rowe MJ, Willis WD, eds. Development, Organization and Processing in Somatosensory Pathways, New York: Alan R Liss Inc 1985; 265-276.

12. Hunkeler W, Möhler H, Pieri L, et al. Selective antagonists of benzodiazepines. Nature 1981; 290: 514-516.

13. Oka J-I, Hicks TP. Benzodiazepines and synaptic processing in the spatial domain within the cat's primary somatosensory cortex. Submitted.

14. Gardiner EP, Costanzo RM. Spatial integration of multiple-point stimuli in primary somatosensory receptive fields of alert monkeys. J Neurophysiol 1980; 43: 420-433.

15. Hicks TP. The history and development of microiontophoresis in experimental neurobiology. Prog Neurobiol 1984; 22: 185-240.

16. Oka J-I, Jang EK, Hicks TP. Benzodiazepine receptor involvement in the control of receptive field size and responsiveness in primary somatosensory cortex. Brain Res 1986; 376: 194-198.

17. Mueller WE, Schlaefer U, Wallert U. Benzodiazepine receptor binding: the interactions of some non-benzodiazepine drugs with specific $\left[{ }^{3} \mathrm{H}\right]$ diazepam binding to rat brain synaptosomal membranes. Naunyn-Schmiedeberg's Arch Pharmacol 1978; 305: 23 26.

18. Mokler DJ, Rech RH. Effects of the benzodiazepine antagonist, Ro 15-1788 on release of conditioned suppression by diazepam, pentobarbital and methaqualone. Pharmacologist 1982; 24: 125.

19. Koe BK, Minor E, Kondratas ME, et al. Enhancement of benzodiazepine binding by methaqualone and related quinazolinones. Drug Devel Res 1986; 7: 255-268.

20. Codding PW, Muir AKS. Molecular structure of Ro 15-1788 and a model for the binding of benzodiazepine receptor ligands. Structural identification of common features in antagonists. Mol Pharmacol 1985; 28: 178-184. 